\title{
Agent Dose Change Type
}

National Cancer Institute

\section{Source}

National Cancer Institute. Agent Dose Change Type. NCI Thesaurus. Code C70892.

An attribute used to characterize a change in the agent dose regimen in the context of its relation to the original documented treatment plan. 